\title{
Bifurcation of Operation Modes of Small Wind Power Stations and Optimization of Their Characteristics
}

\author{
M. Z. Dosaev ${ }^{1 *}$, V. A. Samsonov ${ }^{1 * *}$, Yu. D. Selyutskii ${ }^{1 * * *}$, \\ Wen-Lung $\mathrm{Lu}^{2}$, and Ching-Huei Lin ${ }^{3}$ \\ ${ }^{1}$ Institute of Mechanics, Lomonosov Moscow State University, \\ Michurinskii pr-t 1, Moscow, 119192 Russia \\ ${ }^{2}$ Ching Yun University, \\ 229 Chien-Hsin Road, Jung-Li, Taiwan, 320 Republic of China \\ ${ }^{3}$ National Chiao Tung University, \\ 1001 University Road, Hsinchu, Taiwan, 300 Republic of China \\ Received September 21, 2007
}

\begin{abstract}
Wind stations of small power (small WPS) are intended to ensure the operation of a small number of electric devices. Under these conditions, the connection of even a single additional consumer may result in operation disturbances. In [1,2], a mathematical model of a small WPS operation was proposed, which allowed a qualitative explanation of the energy output hysteresis phenomenon experimentally observed under variation in the external load. In the present paper, on the basis of this model, we study the problems of existence and stability of steady-state modes and describe their attraction domains. We show that there exist different types of bifurcations of these modes, which result, in particular, in the onset of finite-amplitude periodic motions. For the first time, the performance of a power plant is estimated by the maximal consumption power criterion. The results are compared with the results obtained by other criteria.
\end{abstract}

DOI: $10.3103 / \mathrm{S} 002565440902006 \mathrm{X}$

\section{MECHANICAL AND MATHEMATICAL MODEL}

We assume that the wind turbine and the electric generator rotor of a small WPS form a rigid body $G$, which can rotate about a fixed axis in an electromagnetic field created by the generator stator. The position of the body $G$ is determined by its rotation angle $\psi$ (obviously, this is a pseudocyclic coordinate [3]). We denote the body angular velocity by $\omega=\dot{\psi}$. Then the equation of the body rotational motion has the form

$$
J \dot{\omega}=M-c I .
$$

Here $J$ is the moment of inertia of the body $G, M$ is the moment of aerodynamic forces, $I$ is the current in the rotor winding, and $c I$ is the moment created by electromagnetic forces on the rotor axis.

The description of the current dynamics in the circuit depends on the type of the generator and the electric circuit. We restrict ourselves to the simplest case of a direct current generator with independent (constant) excitation, where the external load is an active resistance. Under these conditions, we assume that $c$ is constant and the equation for the current variations has the form

$$
L \dot{I}=c \omega-(R+r) I,
$$

where $L$ is the rotor inductance, $r$ is the rotor internal resistance, and $R$ is the external resistance. The quantity $c \omega$ is the EMF of the rotor inductance.

We assume that flow has a constant velocity $V$ and affects only the blades. As a rule, the shape of the main part of the blade is sufficiently close to the shape of a thin plane plate elongated in the radial

\footnotetext{
*E-mail: maratdz@mail.ru

${ }^{* *}$ E-mail: samson@imec.msu.ru

${ }^{* * * *}$ E-mail: seliytski@imec.msu.ru
} 


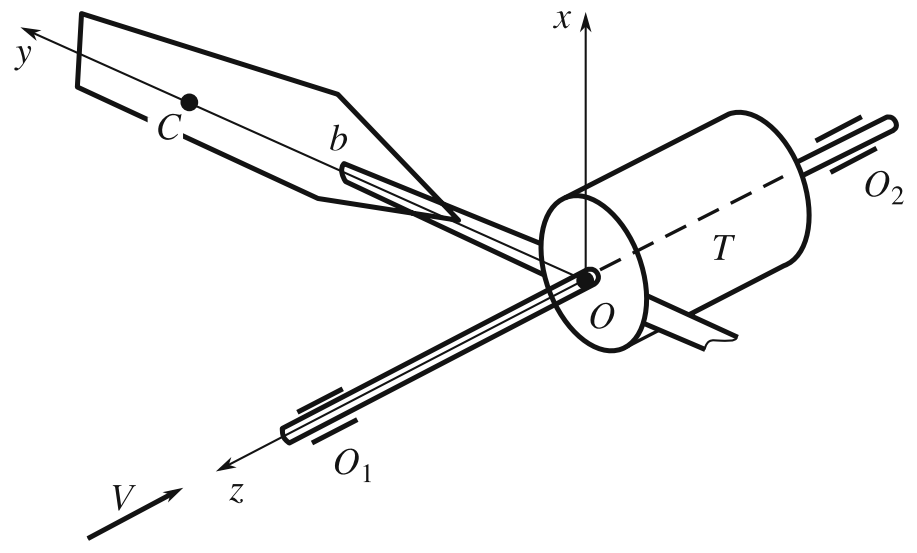

Fig. 1.

direction (the axis $O y$ attached to the body in Fig. 1). Therefore, we can replace the blade by an equivalent plate turned about the axis $O y$ by the setting angle $\varphi$. The zero value of this angle corresponds to the blade position in which the wind turbine does not begin rotating under the wind action (obviously, such a position exists). We assume that the resultant aerodynamic force is applied at a point $C$ of the plate on the axis $O y$ at a distance $b$ from the axis of rotation. The use of such a scheme in the framework of the quasistatic approach was experimentally justified in [4]. In principle, a more detailed description of the flow can be obtained by using methods of applied aerodynamics, but this often requires considerable resources and much more time.

Under the above assumptions, the moment of aerodynamic forces can be represented as

$$
M=\frac{1}{2} \rho S b V_{a}^{2}\left[C_{y}(\alpha) \cos (\varphi-\alpha)-C_{x}(\alpha) \sin (\varphi-\alpha)\right] .
$$

Here $\rho$ is the air density, $S$ is the blade area, $C_{x}(\alpha)$ and $C_{y}(\alpha)$ are the coefficients of drag and bearing power of a separate blade (as a rule, the blades have rather high aerodynamic performance). The instantaneous angle of attack $\alpha$ and the air velocity $V_{a}$ of the pressure center are determined by the relations

$$
\alpha=\varphi-\arctan \left(b \omega V^{-1}\right), \quad V_{a}^{2}=(b \omega)^{2}+V^{2} .
$$

The above model contains several constructive parameters, in particular, $b, \varphi, c, r, J, L$. In general, these parameters must be identified in test experiments for each specific device.

In [1,2], it was shown that, for fixed $b$, the function $M(\omega, \varphi)$ has the following property: for each $\varphi$ in the domain $\omega>0$, there exists a value $\omega_{m}(\varphi)$ of the angular velocity at which the moment attains its maximum. We let $\varphi_{m}$ denote $\omega_{m}\left(\varphi_{m}\right)$ such that

$$
M\left(\omega_{m}\left(\varphi_{m}\right), \varphi_{m}\right)=\max _{\varphi, \omega \geq 0} M(\omega, \varphi)=M^{\max } .
$$

It is necessary to note that for $\varphi \sim 80^{\circ}-90^{\circ}$, the dependencies under study have the following specific property: for relatively small angular velocities, the value of the moment is much less than $M^{\max }$ and can even be negative (i.e., the moment can change from accelerating torque into retarding torque).

\section{STEADY-STATE MOTIONS}

In the case of constant external resistance, the dynamical system (1.1)-(1.2) has fixed points $\left(\omega(t) \equiv \omega_{s}, I(t) \equiv I_{s}\right)$. The corresponding system of equations has the form

$$
\begin{aligned}
& I=\frac{M(\omega, \varphi)}{c}, \\
& I=\frac{c \omega}{R+r} .
\end{aligned}
$$

In Fig. 2, the curve (2.1) is drawn in the phase plane for a certain value of the setting angle. In this case, we used typical aerodynamic characteristics $C_{x}(\alpha), C_{y}(\alpha)$ of elongated plates. The straight 


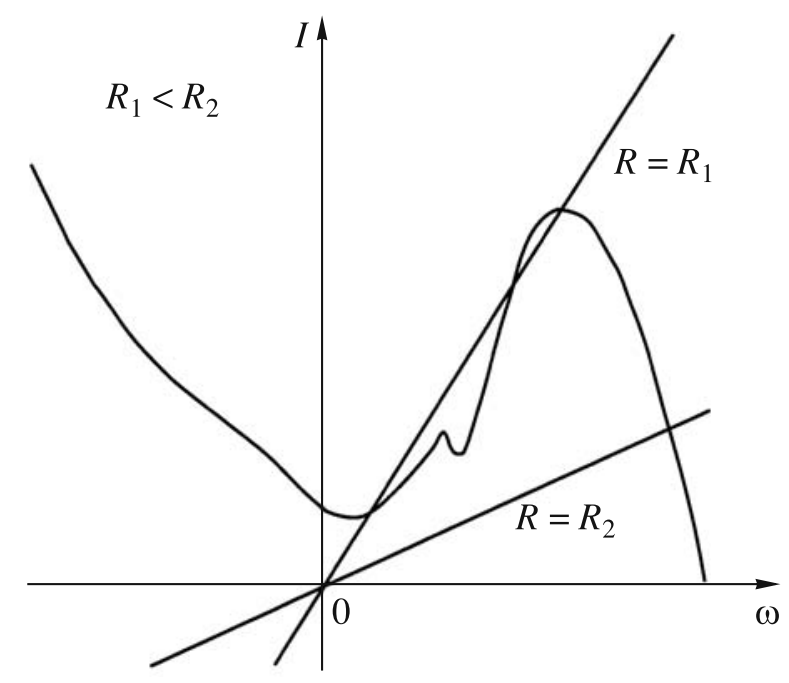

Fig. 2.

lines (2.2) are shown for two values of the external resistance. The points of intersection are fixed points of the system for an appropriate value of $R$. One can see that, for sufficiently large and sufficiently small values of the external resistance, the fixed point is unique. At the same time, there exists a range of values of $R$ in which the system has several fixed points, and this range can be rather wide. The hysteresis phenomenon described in [1] is just related to this fact. Apparently, this phenomenon can disturb the normal operation of the plant.

The fact that there can be several steady-state motions makes the study of their stability rather topical. One can readily show that the stability conditions have the form

$$
\frac{R+r}{L}-\frac{M_{\omega}^{\prime}\left(\omega_{s}, \varphi\right)}{J}>0, \quad \frac{c^{2}}{R+r}-M_{\omega}^{\prime}\left(\omega_{s}, \varphi\right)>0 .
$$

Note that these conditions are necessarily satisfied at the points where $M_{\omega}^{\prime} \leq 0$. Among the fixed points for which the last inequality is not satisfied, there are unstable points. Moreover, a situation is possible in which, for some value of $R$, all steady-state motions occur in the domain $M_{\omega}^{\prime}>0$ and become unstable for a sufficiently large value of the parameter $L$.

Obviously, if there exist several steady-state motions, the motion most preferable as an operation mode is the stable motion with the largest value of the angular velocity (because, in this case, a greater current is produced).

The problem of correspondence between the proposed model and the experimental data was discussed in $[5,6]$. In particular, for a serial wind-driven electric plant "Ar Industrial" of rated power $300 \mathrm{~W}$, several experiments for determining the characteristics of steady-state modes for different values of external resistance were performed in the wind tunnel at the Ching Yun University, This information was used to estimate the values of the parameters $r$ and $c$.

\section{OPTIMIZATION}

The performance of a technical device in the operation mode can be estimated by various criteria. One output characteristic of a wind electric plant (WP) is the torque on the shaft. Therefore, the value of $M^{\max }$ may be of interest for a certain class of problems.

At the same time, in some situations (for example, if the WP is used as a pump drive), the mechanical power taken off by the wind turbine from the flow $N=\omega M(\omega, \varphi)$ may be important. In the literature about the wind-driven electric plants, the problem of maximizing precisely this quantity is discussed.

For any $\varphi$, there exists a value $\omega_{n}(\varphi)$ of angular velocity at which the power attains its maximum. This value is determined by the equation

$$
N_{\omega}^{\prime}=M(\omega, \varphi)+\omega M_{\omega}^{\prime}(\omega, \varphi)=0,
$$

which implies that $\omega_{n}(\varphi)>\omega_{m}(\varphi)$. 


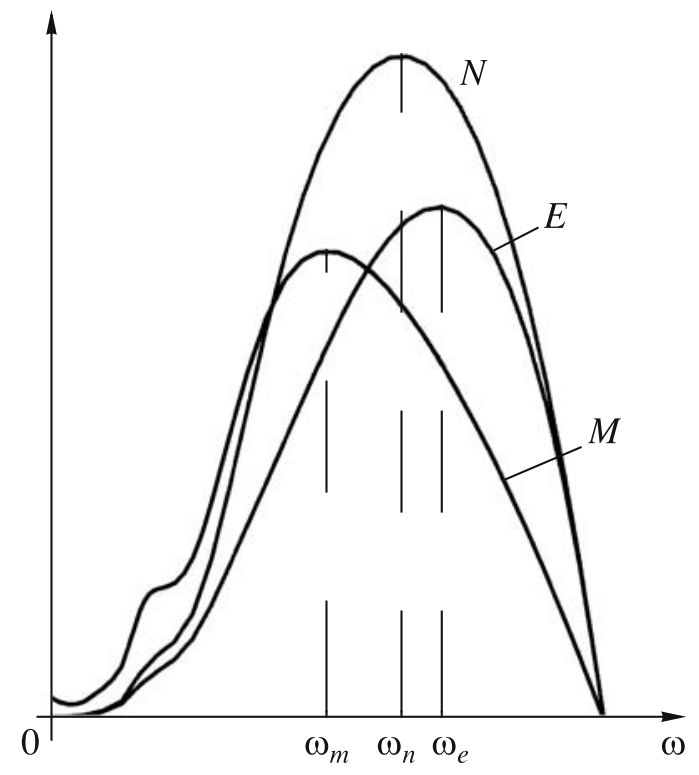

Fig. 3.

But the main characteristic of WPS is its (electric) energy output $E=R I^{2}$. Since the closed model described in $[1,2]$ relates the electric and mechanical characteristics of the plant, it became possible to represent this quantity in the operation mode in the form (using (2.1)-(2.2))

$$
E=\omega M(\omega, \varphi)-r c^{-2} M^{2}(\omega, \varphi) .
$$

The maximum of the function $E$ for a given setting angle is attained for the value $\omega_{e}(\varphi)$ of the angular velocity determined by the equation

$$
E_{\omega}^{\prime}=M(\omega, \varphi)+\omega M_{\omega}^{\prime}(\omega, \varphi)-2 r c^{-2} M(\omega, \varphi) M_{\omega}^{\prime}(\omega, \varphi)=0 .
$$

Obviously, $\omega_{e}(\varphi)>\omega_{n}(\varphi)$ (Fig. 3).

Thus, the optimal design problem for small WPS can be stated in terms of the choice of the setting angle $\varphi_{e}$ and the operation mode $\omega_{e}\left(\varphi_{e}\right)$. Figure 4 displays the curves $E(\omega, \varphi)$ for setting angles in the range $\varphi_{1}<\varphi_{e}<\varphi_{2}$. The external resistance for which the mode of maximal consumption power is realized is determined by formulas $(2.1)-(2.2)$.

\section{TRANSIENT MODES}

The connection or disconnection of a successive consumer (device) to a small WPS is accompanied by a jumpwise variation in the external resistance. Therefore, it is necessary to describe the processes of transition to the new operation mode which arise in this case. This is all the more interesting, because in the case of several stable steady-state motions, each of them has its own attraction domain.

By way of example, consider the case in which the values $R_{0}$ and $\varphi_{0}$ of the external resistance and the setting angle are such that there exist three fixed points $A_{1}\left(\omega_{1}, I_{1}\right), A_{2}\left(\omega_{2}, I_{2}\right)$, and $A_{3}\left(\omega_{3}, I_{3}\right)$, $\omega_{1}>\omega_{2}>\omega_{3}$ (Figs. 5-8), and the condition $M_{\omega}^{\prime}>0$ holds for points $A_{1}$ and $A_{3}$. We note that the fixed point $A_{2}$ is always a saddle point.

The actual generators have a rather small inductance $(L \rightarrow 0)$. The corresponding phase portrait is qualitatively shown in Fig. 5. In this case, the operation mode $A_{1}$ is stable. In Eq. (1.2), there is a small parameter multiplying the highest-order derivative, and we can perform the procedure of separation of motions according to Tikhonov [7]. The fast motions correspond to fast variations in the current for small variations in $\omega$. Therefore, the phase trajectories of fast motions are shown as almost vertical straight lines. The slow motions occur along the straight line (2.2). The directions of motions of the representation point are shown by arrows.

Two separatrix whiskers entering point $A_{2}$ divide the plane into two parts corresponding to the attraction domains of stable operation modes. We note that the origin lies outside the attraction domain 


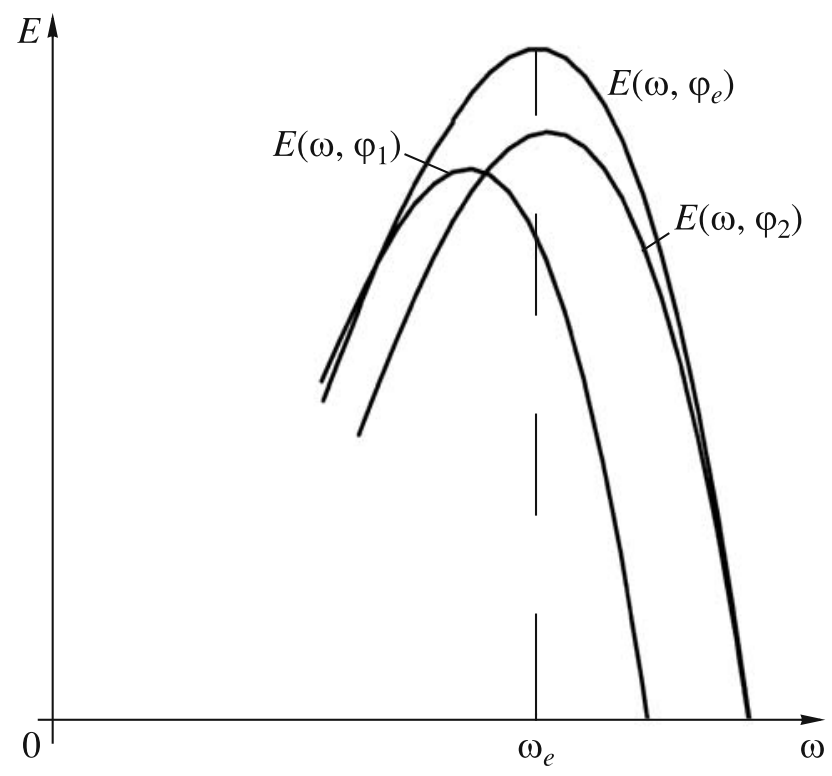

Fig. 4.

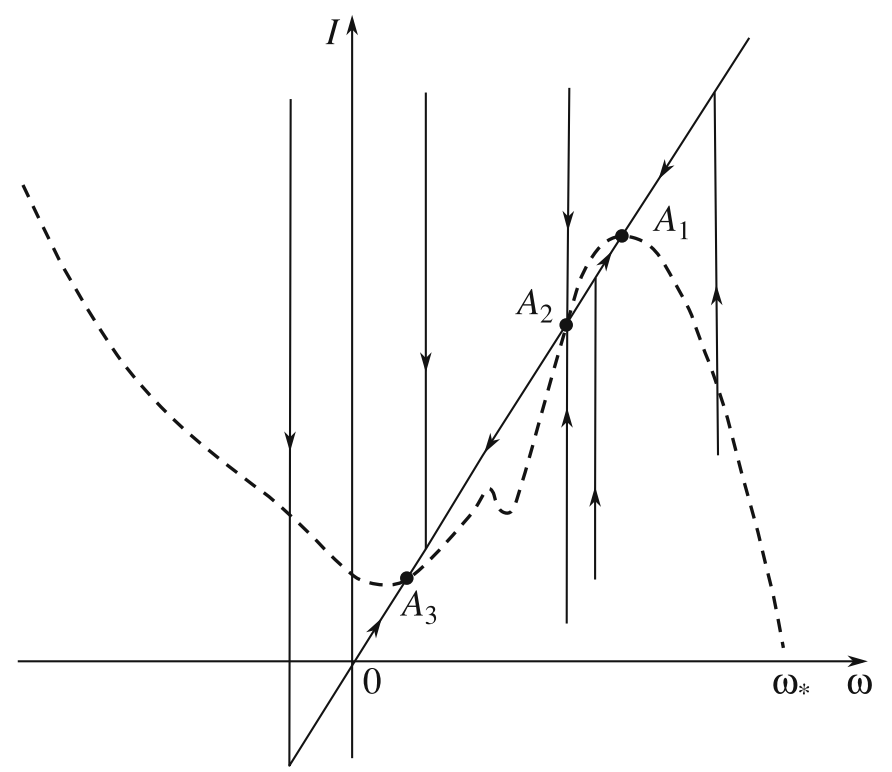

Fig. 5 .

of the preferable operation mode $A_{1}$; i.e., if the generator is switched on at a small angular velocity of the turbine $\left(\omega\left(t_{0}\right) \approx 0, I\left(t_{0}\right)=0\right)$, then the plant is put into an undesirable operation mode. But if the plant functions in the operation mode with angular velocity $\omega>\omega_{2}$, then, in the case of instantaneous decrease in the external resistance (which corresponds to the connection of an additional consumer) to the value $R_{0}$, the plant enters the preferable operation mode $A_{1}$. Indeed, the transient process is sometimes accompanied by overcurrent, i.e., by a sharp increase in the current with its subsequent decrease to the operation value. This overcurrent is the larger the closer the original angular velocity to the angular velocity $\omega_{*}$ of the no-load mode.

In particular, this overcurrent can be decreased by increasing the inductance. Therefore, an analysis of the system behavior for large values of inductance is not only of theoretical but also of practical interest.

First, consider the limit case $L \rightarrow \infty$ (Fig. 6). Note that all fixed points are unstable. In this case, Eq. (1.2) contains a large parameter multiplying the highest-order derivative. Hence $I$ is a slow variable and $\omega$ is a fast variable. The phase trajectories of fast motions are shown by almost horizontal straight 


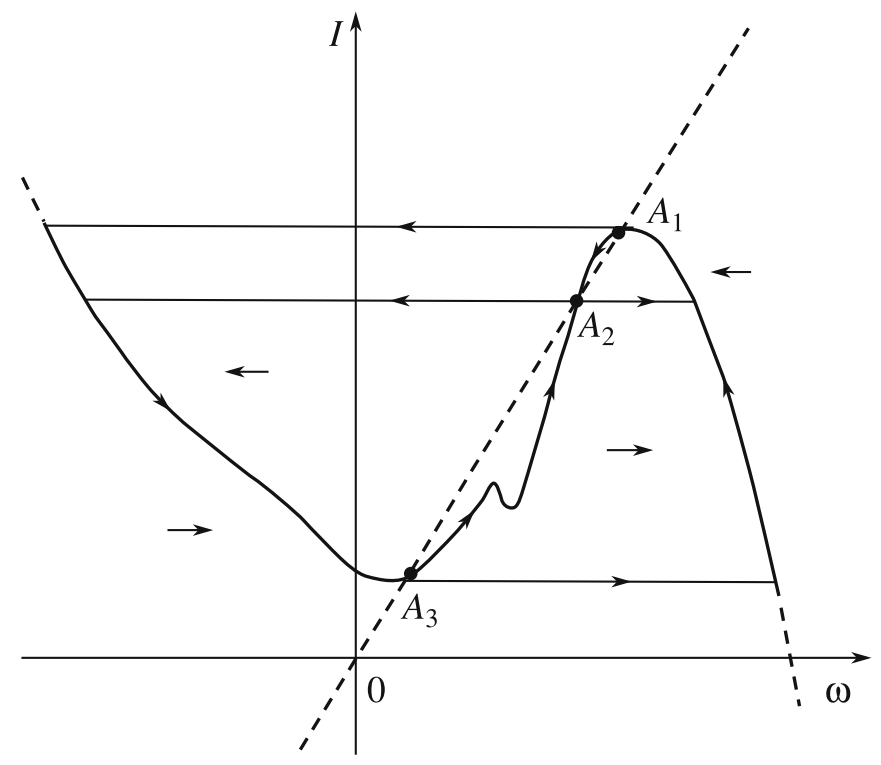

Fig. 6.

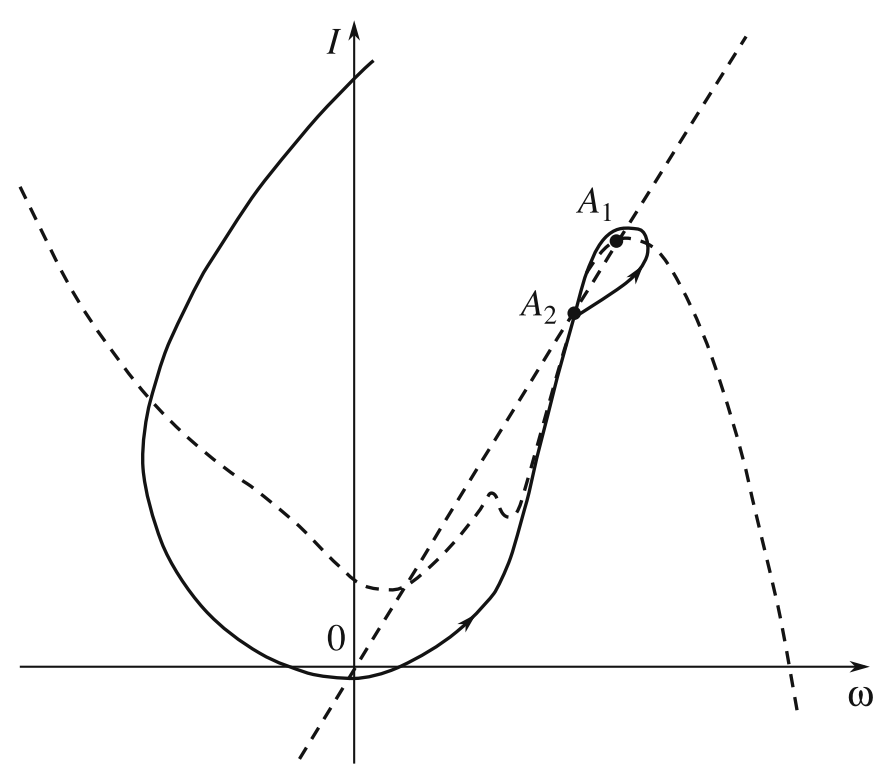

Fig. 7.

lines. The phase trajectories of slow motions are fragments of the curve (2.1). It follows from Fig. 6 that the system has an attracting limit cycle surrounding all fixed points and consisting of fragments of slow motion and fragments of fast motion.

To trace the evolution of stability of fixed points and the reconstruction of the phase portrait structure for varying inductance, we use numerical computations based on the values of the system parameters found in the above-mentioned experiments.

For some $L$, we observe a typical bifurcation of the separatrix loop formation [8], which results in creation of a repulsive limit cycle surrounding the fixed point $A_{1}$ (Fig. 7). As $L$ increases, the attraction domain of the operation mode $A_{1}$ is only the interior of this limit cycle.

The further loss of stability of the fixed point $A_{1}$ is accompanied by the Andronov-Hopf bifurcation caused by the fact that this cycle contracts to a point.

The picture of bifurcations near the steady-state motion $A_{3}$ is more complicated (in particular, there 


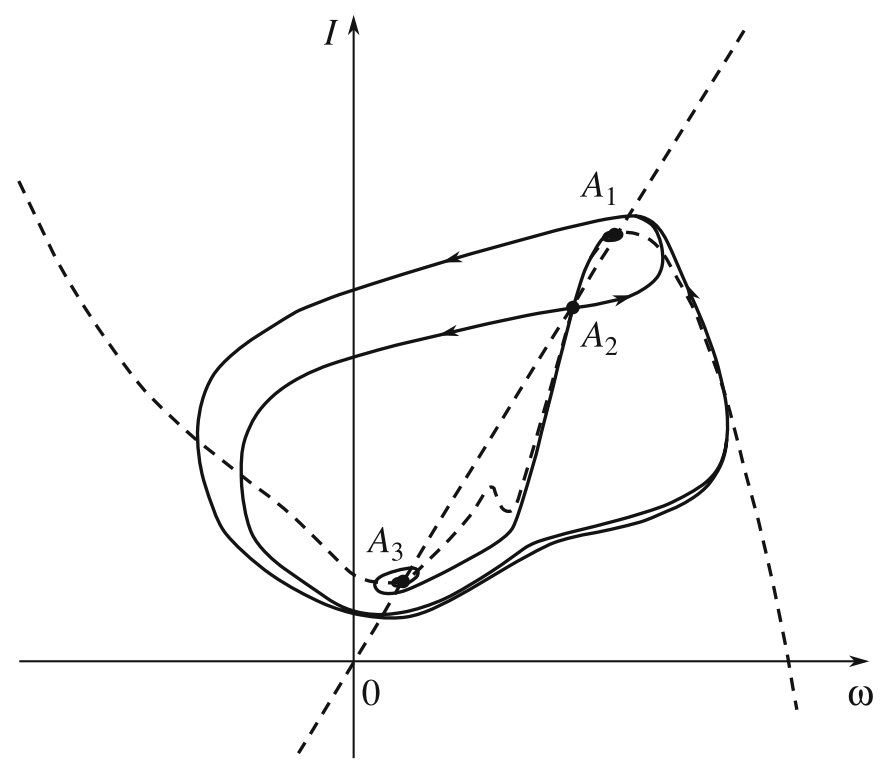

Fig. 8 .

arises a bifurcation of the type of two merging cycles) but, from the practical viewpoint, the study of this part of the phase plane is less interesting.

Note that, in the case of further growth of $L$, all steady-state operation modes cease to be stable, and there appears an attracting limit cycle surrounding all fixed points (Fig. 8). It is curious to note that the motion of the representation point along this periodic trajectory is accompanied by variations in the sign of the angular velocity.

\section{APPROXIMATION OF THE AERODYNAMIC MOMENT}

The aerodynamic moment is a rather complicated nonlinear function (1.3)-(1.4) of the angular velocity. A windwheel whose blades have high aerodynamic performance also has a specific speed, i.e., its operation modes are characterized by the relation $\omega b / V \gg 1$. This fact can be used to construct a simple computational formula for the aerodynamic moment. We illustrate this possibility for the case $\varphi=\pi / 2$. Since the equivalent angle of attack is small under the above assumptions, we obtain

$$
C_{y}(\alpha)=C_{y 1} \alpha-C_{y 2} \alpha^{3}, \quad C_{x}(\alpha)=C_{x 0}+C_{x 2} \alpha^{2},
$$

which implies that

$$
\begin{aligned}
& \frac{M_{a}(\omega, \pi / 2)}{0.5 \rho S b V^{2}}=l-C_{x 0}\left(\frac{\omega b}{V}\right)^{2}-n\left(\frac{V}{\omega b}\right)^{2}, \\
& l=C_{y 1}-C_{x 2}-\frac{C_{x 0}}{2}, \quad n=C_{y 3}-\frac{C_{y 1}}{6}-\frac{C_{x 2}}{6}-\frac{C_{x 0}}{8} .
\end{aligned}
$$

The existence of the last term in (5.1) distinguishes this formula from those usually used in windpower engineering (for example, [9]).

\section{CONCLUSION}

The mechanical and mathematical model proposed above is used to solve the optimization problem for the power consumption, which is the most important output characteristic of a small WPS. A parametric analysis is also performed for the corresponding dynamical system and the bifurcations of different steady-state operation modes are studied for both the steady-state motions and the limit cycles.

\section{ACKNOWLEDGMENTS}

The research was financially supported by the Russian Foundation for Basic Research (projects nos. 08-08-00390 and 09-01-00340) and by the Ching Yun University (Taiwan). 


\section{REFERENCES}

1. M. Z. Dosaev, V. A. Samsonov, Yu. D. Selyutskii, Wen-Lung Lu, and Ching-Huei Lin, "On a Singularity of Operation of Small Wind Power Stations," Vestnik MEI, No. 1, 147-151 (2007).

2. M. Z. Dosaev, V. A. Samsonov, and Yu. D. Selyutskii, "On the Dynamics of a Small-Scale Wind Power Generator,” Dokl. Akad. Nauk 416 (1), 50-53 (2007) [Dokl. Phys. (Engl. Transl.) 52 (9), 493-495 (2007)].

3. V. A. Samsonov, "On the Feasibility of Stabilizing Steady Motions of Systems with Pseudo-Ignorable Coordinates," Prikl. Mat. Mekh. 45 (3), 512-520 (1981) [J. Appl. Math. Mech. (Engl. Transl.) 45 (3), 374-379 (1981)].

4. A. N. Zenkin, V. A. Privalov, and V. A. Samsonov, "Quasi-Static Model of Action of a Medium on an Autorotating Body," Izv. Akad. Nauk. Mekh. Tverd. Tela, No. 4, 73-78 (1993) [Mech. Solids (Engl. Transl.) $28(4), 64-69(1993)]$.

5. Yu. D. Selyutskii, "On Dynamics of Wind Turbines with Horizontal Axis," in Proceedings of the ConferenceCompetition of Young Scientists of Institute of Mechanics of Lomonosov Moscow State University (Izdvo MGU, Moscow, 2007), pp. 47-52.

6. M. Z. Dosaev, L. A. Klimina, B. Ya. Lokshin, et al., "On Stationary Modes of Operation of Wind Turbines," in Proc. IX Intern. Chetaev Conf. "Analytical Mechanics, Stability and Control of Motion," Vol. 2 (Irkutsk, 2007), pp. 69-76.

7. I. V. Novozhilov, Fractional Analysis (Izd-vo MGU, Moscow, 1995) [in Russian].

8. N. N. Bautin and E. A. Leontovich, Methods and Tools for a Qualitative Investigation of Dynamical Systems on Plane (Nauka, Moscow, 1976) [in Russian].

9. V. P. Kharitonov, Autonomous Wind-Driven Electric Plants (Izd-vo GNU VIESKh, Moscow, 2006) [in Russian]. 